\title{
ON LOCALIY DIVIDED INTEGRAL DOMAINS AND CPI-OVERRINGS
}

\author{
DAVID E. DOBBS \\ University of Tennessee \\ Knoxville, Tennessee 37916 \\ U.S.A. \\ (Received March 7, 1979)
}

ABSTRACT. It is proved that an integral domain $R$ is locally divided if and only if each CPI-extension of (in the sense of Boisen and Sheldon) is R-flat (equivalently, if and only if each CPI-extension of $R$ is a localization of R) - Thus, each CPI-extension of a locally divided domain is also locally divided. Treed domains are characterized by the going-down behavior of their CPI-extensions. A new class of (not necessarily treed) domains, called CPIclosed domains, is introduced. Examples include locally divided domains, quasilocal domains of Krull dimension 2, and quasilocal domains with the QQRproperty. The property of being CPI-closed behaves nicely with respect to the $D+M$ construction, but is not a local property.

KEY WOROS AND PHRASES. Integral domain, prime ideal, CPI-extension, flat overring, localization, locally divided, going-down, treed, quasilocal, 22R-property, $\Delta$-domain, D $+M$ construction, Krull dimension. 13AT5, 13C10, 13B99, $13 A 05$. 
1. INTRODUCTION.

In [3], Boisen and Sheldon recently introduced the notion of a CPI-extension of a (commutative integral) domain. For the reader's convenience, we recall the definition of this type of overring and summarize salient results from [3] at the beginning of section 2. Boisen and Sheldon [3, p. 729] have noted that a proper CPI-extension may be integral. Indeed, by combining [6, Corollary 2.6] and [7, Proposition 2.1], it follows that each CPI-extension of a domain $R$ is integral if and only if $R$ is a quasilocal going-down ring, in the sense of [5]. Since a proper integral overring cannot be flat [14, Proposition 2], one might expect a rather different class of domains $R$ to be characterized by the property of having each CPI-extension of $R$ being $R$-flat. The main result of section 2, Theorem 2.4, establishes that the domains thus characterized are the locally divided domains introduced in [6]. Prüfer domains are perhaps the most natural examples of locally divided domains. It was shown in [6] that any locally divided domain is a (not necessarily quasilocal) going-down ring, and that the converse holds in the root-closed case. As byproducts, Proposition 2.3 establishes that a CPI-extension $T$ of a domain $R$ is R-flat (if and) only if $T$ is a localization (in the sense of "ring of fractions" in [4, p. 57]) of $R$; and Corollary 2.6 establishes that each CPI-extension of a locally divided domain is also locally divided. The final result in section 2 shows how going-down behavior of CPI-extensions serves to characterize treed domains.

Divided domains are precisely the domains which coincide with each of their CPI-extensions. More generally, section 3 is devoted to studying domains $R$ which are "CPI-closed," in the sense that each CPI-extension of a CPI-extension of $R$ is itself a CPI-extension of $R$. (A more useful characterization of CPI-closed domains is given in Proposition 3.2.) Examples of CPI-closed domains include the locally divided domains; the quasilocal domains of Krull dimension 2; 
and quasilocal domains with the QQR-property (see Corollary 3.3, Remark 3.5 and Proposition 3.7, respectively). Despite expectations raised by the first-cited example, the second family of examples illustrates that a CPI-closed domain need not be treed. The quasilocal, treed CPI-closed domains are characterized in Proposition 3.9 and Remark 3.10(a). As shown by Example 3.6, being CPI-closed is not a local property, since the ring of polynomials in two variables over a field is not CPI-closed. Examples of non-CPI-closed domains with arbitrary Krull dimension exceeding 1 then result from the $D+M$-construction (see Proposition 3.11). In Remarks 3.8 and $3.10(\mathrm{~b})$, (c), we raise some open questions relating divided domains, domains with the QQR-property, certain types of CPIclosed domains, and the $\Delta$-domains of Gilmer and Huckaba [10].

\section{LOCALLY DIVIDED DOMAINS.}

As defined by Boisen and Sheldon [3], a CPI-extension of the domain $R$ is an overring of $R$ of the form $R+P R_{P}$, for some prime ideal $P$ of $R$. The terminology "CPI" stands for "complete pre-image," and is well chosen inasmuch as $R+P_{P}$ is easily shown to be (canonically isomorphic to) the pullback, in the category of commutative rings with unit, of the diagram

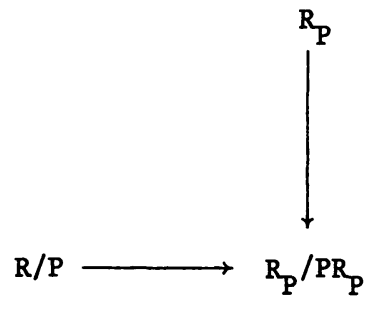

where the vertical map is the canonical surjection and the horizontal map is the inclusion. Reasoning as in [11, Remark 3.9], we find that applying the contravariant functor Spec to the above diagram produces a pushout diagram in the 
(dual) category of affine schemes. In particular, $S p e c\left(R+P R_{P}\right)$ may be viewed set-theoretically as the quotient space of the disjoint union of $\operatorname{Spec}\left(R_{\mathrm{P}}\right)$ and $\operatorname{Spec}(R / P)$ in which the prime $P_{P}$ of $R_{P}$ is identified with the zero prime $\mathrm{P} / \mathrm{P}$ of $\mathrm{R} / \mathrm{P}$. We next summarize some more precise order-theoretic information gleaned from [3, Section 2]. The method used in the proof of [6, Lemma 2.4] to study the case in which $R+P_{P}$ is integral over $R$ also extends to yield the same facts.

LEMMA 2.1. (Boisen and Sheldon). Let $T=R+P R_{P}$ be the CPI-extension of the domain $R$ corresponding to the prime $P$ of $R$. Then the contraction map $\operatorname{Spec}(T) \rightarrow \operatorname{Spec}(R)$ sets up an isomorphism of partially ordered sets (with respect to inclusion) between $\operatorname{Spec}(T)$ and the set of primes of $R$ which are comparable to $P$. If $Q$ is a prime of $R$ which is contained in $P$, then $Q$ corresponds to $Q_{P} \in \operatorname{Spec}(T)$. If $Q$ is a prime of $R$ which contains $P$, then $Q$ corresponds to $Q+\mathrm{PR}_{\mathrm{P}} \in \operatorname{Spec}(\mathrm{T})$.

The next result collects some facts which will be used below.

LEMMA 2.2. Let $P$ and $Q$ be comparable primes of the domain $R$. Let $T$ be the CPI-extension of $R$ with respect to $P$, i.e., $T=R+P R_{P}$. Then:

(a) If $Q \subset P$, then $T_{Q_{P}}=R_{Q}$.

(b) If $P \subset Q$, then $T_{R Q}=R_{Q}+P R_{P}=T_{Q}+P R_{P}$.

PROOF. (a): As noted in Lemma 2.1, $Q_{P} \cap R=Q$ if $Q \subset P$. Hence, $R_{Q} \subset T_{Q R_{P}}$. For the reverse inclusion, observe $T \subset R_{P}$, so that $T_{Q_{P}} \subset\left(R_{P}\right)_{Q R_{P}}=R_{Q}$, the final step coming from [8, Corollary 4.3].

(b): Let $P \subset Q$. As the inclusions $T_{R \backslash Q} \subset T_{Q}+P_{P}$ and $R_{Q}+P R_{P} \subset T_{R \backslash Q}$ are obvious, it remains only to prove that $T_{Q}+P_{P} \subset R_{Q}+P R_{P}$. To this end, consider a typical element $x$ of the left hand side. Now, $x=t\left(r+p z^{-1}\right)^{-1}$ for some $t \in T, r \in R \backslash Q, P \in P$ and $z \in R \backslash P$. As $T=R+P R_{P}$ and $P_{P}$ 
Is the set of nonunits of $R_{P}$, it follows that, in order to prove $x \in R_{Q}+P R_{P}$, we may assume $t \in R$. A short calculation reveals that $x=\operatorname{tr}^{-1}+y$, when $y=-p t r^{-1}(r z+p)^{-1}$. Since $\operatorname{tr}^{-1} \in R_{Q}$ and $y \in P R_{P}$, the proof is complete. Before presenting the main result of this section (Theorem 2.4), we isolate a fragment which is of some independent interest.

PROPOSITION 2.3. Let $T=R+P R_{P}$ be the CPI-extension of the domain $R$ corresponding to the prime $P$ of $R$. Then $T$ is a flat R-module if and only if $T$ is a localization of $R$.

PROOF. The "If" half is immediate, as any localization is flat [4, Theorem 1 , p. 68]. Conversely, let $T$ be R-flat. As any domain (in particular, $T$ ) is the intersection of its localizations at maximal ideals and the maximal ideals of $T$ are classified in Lemma 2.1, [14, Theorem 2] yields $T=R_{M}$, where $M$ ranges over the set of maximal ideals of $R$ which contain $P$. If $S=R \backslash(U M)$, it is clear that $R_{S} \subset T$, and so it suffices to prove that $T \subset R_{S}$.

To that end, let $t \in T$, and consider the conductor $I=\{x \in R: x t \in R\}$. Note that, for each maximal ideal $M$ of $R$ which contains $P$, we have $I \notin M$ since $T \subset R_{M}$. If $t \notin R_{S}$, then $I \cap S=\phi$ and, by Zorn's lemma, $I$ is contained in a prime ideal $N$ which is also disjoint from $S$. If $P+N$ were contained in a maximal ideal $Q$ of $R$, then $Q$ would be an $M$ of the above type (since $P \subset Q$ ), whence $I \notin Q$ by the above argument, although $I \subset N \subset Q$. Consequently, $P+N=R$, and so $p+n=1$, for some $p \in P$ and $n \in N$. Since $N \subset R \backslash S$, there exists a maximal ideal $W$ of $R$ such that $P \subset W$ and $\mathbf{n} \in \mathbf{W}$. Then

$$
1=p+n \in P+W \subset W+W=W
$$

the desired contradiction, to complete the proof.

Recall from [6] that a domain $R$ is said to be divided in case $P=P R_{P}$ 
for each prime ideal $P$ of $R$. (This notion was introduced in [1] by Akiba who termed such objects "AV-domains.") Any divided domain is quasilocal (cf. [1, Theorem 1]). A domain $R$ is called locally divided if $R_{M}$ is divided for each maximal ideal $M$ of $R$ (equivalently, by [6, Remark 2.7(b)], for each prime $M$ of $R$ ). As noted in [6, Remark 2.7(b)], any localization of a locally divided domain is itself locally divided; and any locally divided domain is a going-down ring, in the sense of [5, p. 448]. (While the converse of the preceding assertion is false [6, Example 2.9], any root-closed going-down ring must be a locally divided domain [6, Corollary 2.8].) Thus, by either [1, Theorem 1] or [5, Theorem 2.2], any locally divided domain $R$ is treed, in the sense that no maximal ideal of $R$ contains incomparable prime ideals.

THEOREM 2.4. For any domain $R$, the following six conditions are equivalent:

(1) $R$ is locally divided;

(2) For each prime $P$ of $R$, the CPI-extension $R+P R_{P}$ is $R-f l a t ;$

(3) For each prime $P$ of $R$, the CPI-extension $R+P R_{P}$ is a localization of $R$;

(4) For any comparable primes $P \subset Q$ of $R$, the corresponding CPI-extensions satisfy $R+\mathrm{PR}_{\mathrm{P}} \subset \mathrm{R}+\mathrm{QR}_{\mathrm{Q}}$;

(5) For any comparable primes $\mathrm{P} \subset \mathrm{Q}$ of $\mathrm{R}$, the containment $\mathrm{PR}_{\mathrm{P}} \subset \mathrm{PR}_{\mathrm{Q}}$ holds;

(6) For any comparable primes $P \subset Q$ of $R$, the containment $P R_{P} \subset R_{Q}$ holds.

PROOF. (5) $\Leftrightarrow(1)$ : Establishing (1), i.e., that $R_{Q}$ is a divided domain for each maximal (equivalently, for each prime) ideal $Q$ of $R$, amounts to showing, for each prime $P$ of $R$ which is contained in $Q$, that ${ }_{P R}$ coincides with $\left(P R_{Q}\right)\left(R_{Q}\right)_{P R_{Q}}$. By the result cited in the proof of Lemma 2.2 (a), the latter prime is just $\left(P R_{Q}\right) R_{P}$ which, since $R_{Q} \subset R_{P}$, simplifies to $P_{P}$. 
As $P \subset Q$ also forces $P R_{Q} \subset P R_{P}$, it is now clear that (5) $\Leftrightarrow$ (1).

(1) $\Rightarrow(2)$ : Let $T=R+P_{P}$ for some prime $P$ of $R$. To show that $T$ is R-flat, it is enough, by [4, Proposition 15, p. 91], to establish that $T_{R \backslash M}$ is $R_{M}-f$ lat for each maximal ideal $M$ of $R$. If $P \subset M$, then Lemma 2.2(b) gives $T_{R \backslash M}=R_{M}+P R_{P}$, while the hypothesis (1) [with the aid of the implication $(1) \Rightarrow(5)$ established above] leads to $P R_{P}=P R_{M}$, so that $T_{R \backslash M}=R_{M}+P R_{M}=R_{M}$ which is, indeed, $R_{M}$-flat. Finally, in case $P \notin M$, the hypothesis (1) is not needed:

$$
T_{R \backslash M}=R_{M}+P R_{P} R_{M}=R_{M}+R_{M} R_{P}=R_{M} R_{P}=\left(R_{M}\right)_{R \backslash P},
$$

the second equality arising since $P_{M}=R_{M}$. Thus, if $P \not \subset M$, then $T_{R \backslash M}$ is a localization of (and, hence, is flat over) $R_{M}$.

(2) $\Leftrightarrow$ (3): Apply Proposition 2.3.

(2) $\Rightarrow(4)$ : Assume, by (2), that the primes $P \subset Q$ of $R$ induce $R-f l a t$ CPI-extensions $S=R+P R_{P}$ and $T=R+Q R_{Q}$. As in the proof of Proposition 2.3, $S=R_{M}$ and $T=R_{N}$ where $M$ (resp., $N$ ) ranges over the set of maximal ideals of $R$ which contain $P$ (resp., $Q$ ). Since $P \subset Q$, each ideal (index) of the form $N$ is also of type $M$, whence $S \subset T$, as desired.

Finally, observe that the implications $(4) \Rightarrow(6)$ and $(6) \Rightarrow(5)$ are straightforward, and the proof is complete.

The remainder of this section analyzes further the nonreversible implications

locally divided domain $\Rightarrow$ going-down ring $\Rightarrow$ treed domain.

(The simplest known example of a treed domain which is not a going-down ring is due to W.J. Lewis and appears in [12, Example 6.4].) As motivation for the next result, note that the examples in [5, Corollary 4.4(ii)] combine with 
[6, Lemma 2.2(b)] to show that, for each positive integer $n$, there exists a divided domain which has Krull dimension $\mathrm{n}$ and possesses an overring which is not treed.

PROPOSITION 2.5. Let $T$ be an R-flat overring of a domain $R$. If $R$ is locally divided (resp., a going-down ring; resp., treed), then $T$ has the corresponding property.

PROOF. It suffices to prove that $T_{N}$ has the appropriate property for each maximal ideal $N$ of $T$. Let $M=N \cap R$. As $R_{M}$ inherits the appropriate property from $R$ and $R$-flatness of $T$ forces $R_{M}=T_{N}$ [14, Theorem 2], the proof is complete.

COROLLARY 2.6. Let $T=R+P R_{P}$ be the CPI-extension of a domain $R$ arising from a prime $P$ of $R$. If $R$ is locally divided (resp., a goingdown ring; resp., treed), then $T$ has the corresponding property.

PROOF. If $R$ is locally divided, then the implication $(1) \Rightarrow$ (2) in Theorem 2.4 assures that $T$ is R-flat, and so Proposition 2.5 applies, showing that $T$ is also locally divided.

The assertions about going-down rings and treed domains are not corollaries of Proposition 2.5, but are instead simple consequences of Lemma 2.1. Details may be safely omitted.

It should be noted that it is possible to give a proof of the assertion in Corollary 2.6 concerning locally divided domains without recourse to the notion of flatness. Indeed, one may show that $T$ inherits from $R$ the property of satisfying condition (5) in Theorem 2.4. There are three cases to consider, determined by the nature of the containments between the prime defining $T$ and the primes figuring in (5). Each case is treated with the aid of appropriate parts of Lemma 2.2. Details are left to the reader. 
In view of the example of Lewis mentioned above, it is somewhat interesting that treed domains may be characterized by appropriate going-down phenomena: as Proposition 2.7 indicates, the CPI-overrings hold the key. Another consequence of Proposition 2.7 is that, if the "flat" property in condition (2) of Theorem 2.4 is weakened to the "going-down" property, then the class of domains thereby chatacterized is enlarged from the locally divided domains to the treed domains. Finally, we note that $(2) \Rightarrow(1)$ in Proposition 2.7 may be viewed as a generalization of [1, Theorem 1].

PROPOSITION 2.7. For any domain $R$, the following are equivalent:

(1) $\mathrm{R}$ is treed;

(2) $\mathrm{R} \subset \mathrm{T}$ has the going-down property for each CPI-extension $T$ of $R$. PROOF. (1) $\Rightarrow$ (2). Suppose that $T=R+P R_{P}$ for some prime $P$ of $R$. Verifying that $R \subset T$ has the going-down property amounts to showing that whenever $P_{2} \subset P_{1}$ are primes of $R$ and $Q_{1}$ is a prime of $T$ such that $Q_{1} \cap R=P_{1}$, then there exists a prime $Q_{2}$ of $T$ such that both $Q_{2} \subset Q_{1}$ and $Q_{2} \cap R=P_{2}$. Given $Q_{1}$, we infer from Lemma 2.1 that $P_{1}$ is comparable to $P$. To produce a satisfactory $Q_{2}$ via the recipe in Lemma 2.1 , it is enough to show that $P_{2}$ is also comparable to $P$. As $P_{2} \subset P_{1}$, we may suppose that $P_{1} \not P$, in which case $P \subset P_{1}$. Then, if $R$ is treed, $P_{2}$ and $P$ must be comparable, for they both lie within (any maximal ideal containing) $\mathrm{P}_{1}$

$(2) \Rightarrow(1)$ : Let $P$ and $Q$ be prime ideals of $R$ which are each contained in a given maximal ideal $M$ of $R$. Let $T=R+P R_{P}$, the CPI-extension of $R$ with respect to $P$. Recall that $Q_{1}=M+P R_{P}$ is a prime ideal of $T$ such that $Q_{1} \cap R=M$. As $Q \subset M$, the requirement that $R \subset T$ have the going-down property produces a prime $Q_{2}$ of $T$ such that $Q_{2} \cap R=Q \quad$ (and $Q_{2} \subset Q_{1}$ ). Since $Q$ is in the image of the contraction map $\operatorname{Spec}(T) \rightarrow \operatorname{Spec}(R)$, Lemma 2.1 
yields the comparability of $Q$ and $P$, to complete the proof.

\section{CPI-CLOSED DOMAINS.}

The definition of this section's main object of study is motivated by the observation that it is possible to iterate the process of taking CPI-extensions. More precisely, we shall say that a domain $R$ is CPI-closed if each CPI-extension of each CPI-extension of $R$ is itself a CPI-extension of $R$. Trivally, any divided domain is CPI-closed. Indeed, so is any locally divided domain, but the proof of this and the furnishing of more examples must await the next two results.

LEMMA 3.1. Let $P$ and $Q$ be comparable primes of a domain $R$, and let $T=R+P R_{P}$, the CPI-extension of $R$ with respect to $P$. Let $W$ be the prime of $T$ such that $W \cap R=Q$. (By Lemma 2.1, $W=Q_{P}$ if $Q \subset P$, and $W=Q+P R_{P}$

if $\left.P \subset Q_{.}\right)$Then the CPI-extension of $T$ with respect to $W$ is $R+P_{P}+Q_{Q}$. PROOF. One need merely simplify $T+W_{W}$ with the help of the appropriate part of Lemma 2.2. Details are left to the reader.

As an immediate consequence, we have:

PROPOSITION 3.2. A domain $R$ is CPI-closed if and only if, whenever $P$ and $Q$ are comparable primes of $R$, the sum of the CPI-extension of $R$ with respect to $P$ and the CPI-extension of $R$ with respect to $Q$ is itself a CPIextension of $R$.

COROLLARY 3.3. Any locally divided domain is CPI-closed.

PROOF. Observe that condition (4) of Theorem 2.4 implies the criterion given in Proposition 3.2.

Similar reasoning also establishes the next result.

COROLLARY 3.4. If a domain $R$ is such that the set of CPI-extensions of $R$ is totally ordered with respect to inclusion, then $R$ is CPI-closed.

As usual, $\operatorname{dim}(R)$ will denote the Krull dimension of $R$. 
REMARK 3.5. Each of the following two conditions is sufficient for a domain R to be CPI-closed:

(a) $\operatorname{dim}(R) \leq 1$;

(b) $R$ is quasilocal and $\operatorname{dim}(R)=2$.

Accordingly, a CPI-closed domain need not be treed; a treed CPI-closed domain need not be a going-down ring; and a CPI-closed going-down ring need not be locally divided.

PROOF. The fact that (a) and (b) are each sufficient follows, as the criterIon in Proposition 3.2 is easily seen to be satisfied in these cases. (Moreover, (a) is a special case of Corollary 3.3.) As for the final assertion; consider the (quasilocal) nontreed domain $R$ in [13, Example 2.28]; the (quasilocal) example of Lewis [12, Example 6.4] of a treed domain which is not a going-down ring; and the (quasilocal) going-down ring which is not a divided domain, constructed by Boisen and Sheldon [2, Example 1.6] and studied further in [6, Example 2.9]. By (b), each of these three rings is CPI-closed, completing the proof.

We pause to present an example of a domain which 18 not CPI-closed. By combining Remark 3.5(b) and the observations to be made in Example 3.6 , we shall see that a domain $R$ need not be CPI-closed even when $R_{P}$ is CPI-closed for each prime $P$ of $R$.

EXAMPLE 3.6. Let $\mathrm{X}$ and $\mathrm{Y}$ be algebraically independent indeterminates over a field $\mathrm{K}$. Then the polynomial ring $\mathrm{R}=\mathrm{K}[\mathrm{X}, \mathrm{Y}]$ is not CPI-closed. PROOF. Deny. Then, if we consider the comparable primes $P=X R$ and $Q=X R+Y R$, Proposition 3.2 supplies a prime $W$ so that

$$
R+P R_{P}+Q R_{Q}=R+W R_{W}
$$

As $W R_{W} \cap\left(R+P R_{P}\right)$ is a prime of $R+P R_{P}$ whose intersection with $R$ is $W$, Lemma 2.1 shows that $W$ and $P$ are comparable. Similarly, $W$ and $Q$ are 
comparable. Thus, since $Q$ is maximal and has height 2 , either $W=P$ or $W=Q$.

Assume that $\mathrm{W}=\mathrm{P}$. Then

$$
\mathrm{Y}(1+\mathrm{Y}+\mathrm{X})^{-1}=\mathrm{f}+\mathrm{Xgh}^{-1}
$$

for some elements $f, g$ and $h$ of $R$ such that $h \notin W$. Crossmultiply and substitute $X=0$. If $F_{0}$ denotes the result of substituting $X=0$ into a polynomial $F$, the result may be written as

$$
h_{0} Y=f_{0} h_{0}(1+Y) \text {. }
$$

Since $h \notin W$, we have $h_{0} \neq 0$, and so cancellation yields $Y=f_{0}(1+Y)$, a contradiction since $R$ is a unique factorization domain.

The resulting case, $W=Q$, is handled similarly. Write

$$
\mathrm{XY}^{-1}=\mathrm{f}+\mathrm{we}^{-1}
$$

for some $f \in R, w \in W$ and $e \in R \backslash W$. Multiply by Ye and then set $Y=0$. If $e_{0}$ results from $e$ by setting $Y=0$, we thus obtain $e_{0} X=0$, whence $e_{0}=0$ and $e \in Y R \subset W$, the desired contradiction. This completes the proof. Before presenting another family of CPI-closed domains, we recall from [9] that a domain $R$ is said to have the $Q Q R$-property in case each overring of $R$ is an intersection of localizations of $R$. It is known that a domain $R$ is a Prüfer domain if and only if $R$ is integrally closed and has the $Q Q R$-property [9, Corollary 1.7]. There exist (quasilocal) domains with the QQR-property which are not Prüfer domains [9, Example 4.3]; by a result of Davis (cf. also [9, Theorem 1.4]), any such domain $R$ satisfies $\operatorname{dim}(R)=\infty$.

PROPOSITION 3.7. Let $R$ be a quasilocal domain with the QQR-property. Then $R$ is CPI-closed. Indeed, if $P$ and $Q$ are comparable primes of $R$ such that 
$\mathrm{PR}_{\mathrm{P}} \neq \mathrm{P}$ and $\mathrm{QR} \mathrm{Q}_{\mathrm{Q}} \neq \mathrm{Q}$, then $\mathrm{R}+\mathrm{PR}_{\mathrm{P}}=\mathrm{R}+\mathrm{QR}_{\mathrm{Q}}$.

PROOF. The proof consists of applying some results of Gilmer and Heinzer [9]. Since valuation domains, being divided, are CPI-closed, we may suppose that $R$ is not a valuation domain. Then $R^{\prime}$, the integral closure of $R$, is a Prüfer domain [9, Corollary 1.7]; and $R^{\prime}$ is contained in each proper overring of $R$ [9, Theorem 1.10]. In view of Proposition 3.2, it suffices to establish the final assertion, i.e., that $R+P R_{P}=R+Q R_{Q}$ under the stated conditions. Observe that $A=R+P R_{P}$ is a valuation domain, for it is quasilocal (by Lemma 2.1) and it is an overring of the Prüfer domain $R^{\prime}$. As $B=R+P R_{P}+Q_{Q}$ is a CPI-extension of $A$ (by Lemma 3.1) and $A$ is divided, it follows that $A=B$ and, in particular, $R+Q R_{Q} \subset A$. To establish the reverse inclusion, reverse the roles of $P$ and $Q$ in the preceding argument. This completes the proof.

REMARK 3.8. We have seen that the class of valuation domains may be properly extended in (at least) two ways: to the class of divided domains, and to the class of quasilocal domains having the $Q Q R$-property. It is easily seen that the former of these "extended classes" is not contained in the latter. Indeed, Gilmer and Huckaba [10, Example 3] construct a quasilocal domain $D$ which does not have the QQR-property, although [6, Lemma 2.2(b)] reveals that $D$ is divided. As for the reverse (non)containment, we do not know of a quasilocal domain with the $Q Q R$-property which is not divided (but we suspect that such exists). In any event, it should be noted that the class of quasilocal CPI-closed domains properly contains the "union" of the two "extended classes," as the result of [9] quoted just prior to the statement of Proposition 3.7 shows that the final example in Remark 3.5, which has already been seen to be a quasilocal nondivided CPI-closed domain, also falls to have the $Q Q R$-property.

While it was natural in section 2 to consider embedding the class of locally divided domains in the class of treed domains, Example 3.6 reveals that, in 
passing from the class of locally divided domains to the larger class of CPI-closed domains, some nontreed domains (e.g., $\mathrm{K}[\mathrm{X}, \mathrm{Y}]$ ) have been introduced. The next result interposes a class of quasilocal treed domains properly between the divided domains and the CPI-closed domains, and incidentally generalizes Corollary 3.4.

PROPOSITION 3.9. Let the domain $R$ be such that each sum of two CPI-extensions of $R$ is itself a CPI-extension of $R$. Then $R$ is CPI-closed, quasilocal and treed.

PROOF. Proposition 3.2's criterion clearly holds, and so $R$ is CPI-closed. It remains only to show that any two primes of $R$, say $M$ and $N$, are comparable. Suppose, instead, that $M \not \subset N$ and $N \not M$. By hypothesis, $R+M_{M}+N_{N}$ is a ring, in fact of the form $R+P_{P}$ for some prime $P$ of $R$. The incomparability assumptions give $\mathbb{R R}_{N}=R_{N}$ and $\mathbb{R R}_{M}=R_{M}$, respectively, so that $\left(\mathbb{R R}_{M}\right)\left(\mathrm{NR}_{N}\right)=\mathrm{R}_{M} R_{N}$. Thus, $R+P R_{P}=R_{S}$, where $S=\{a b \in R: a \in R \backslash M, b \in R \backslash N\}$. Since $P R_{P}$ is a prime of $R_{S}$ which intersects $R$ in $P$, we have $P \cap S=\emptyset$, and so $P \subset M \cap N$. Since $P$ and $M$ are therefore comparable, Lemma 2.1 implies that some prime of $R_{S}$ intersects $R$ in $M$, whence $M \cap S=\emptyset$. However, $M \cap S \supset M \cap(R \backslash N) \neq \emptyset$, the desired contradiction.

REMARK 3.10. (a) It is clear from Proposition 3.2 that the converse of Proposition 3.9 is also valid.

(b) The second of the three examples in Remark 3.5 shows that the "treed" conclusion in Proposition 3.9 cannot be strengthened to "going-down ring," and hence certainly cannot be strengthened to "divided." Note that this example satisfies the hypothesis of Proposition 3.9 since it satisfies the ostensibly more stringent assumption of Corollary 3.4. We say "ostensibly," for we do not know (but doubt) whether, conversely, "the sum of any two CPI-extensions of the domain $R$ is itself a CPI-extension of $R^{\prime \prime}$ implies "the set of CPI-extensions of $R$ is totally ordered by inclusion." of course, the implication does hold 
if $\operatorname{dim}(R) \leq 3$.

(c) Despite (b), one can strengthen the hypothesis in Corollary 3.4 in order to get a "going-down" conclusion. Indeed, Papick [12, Lemma 2.41] has shown, with the aid of [10, Theorem 4], that if the set of all the overrings of a domain $R$ is totally ordered by inclusion, then $R$ is a quasilocal 1 -domain (in the terminology of [13]) and, hence, $R$ is a going-down ring. The crucial point is that such an $R$ is a $\Delta$-domain. (Following Gilmer and Huckaba [10], we say that a domain $R$ is a $\Delta$-domain in case each sum of two overrings of $R$ is a ring.) It should be noted that a quasilocal 1 -domain need not have its overrings forming a totally ordered set, since [10, Example 4] presents a quasilocal 1-domain which is not a $\Delta$-domain; and $[10$, Propositions 10 and 11$]$ exhibits a $\Delta$-domain whose set of overrings fails to be totally ordered.

Apart from the semantic similarity between the criterion in Proposition 3.2 and the definition of $\Delta$-domain, one should note that any quasilocal $Q Q R$-domain is both CPI-closed and a $\Delta$-domain (by Proposition 3.7 and [10, Theorem 5]). We do not know of a $\Delta$-domain which is not CPI-closed. However, a CPI-closed domain need not be a $\Delta$-domain; indeed, a divided domain need not be a $\Delta$-domain. For a quasilocal example of Krull dimension 1, use efther the ring $D_{6}$ in [10, Proposition 10] or the ring $D$ in [10, Example 4]; an Integrally closed example may be fashioned from [5, Theorem 4.2], by recalling that any integrally closed $\Delta$-domain must be a Prüfer domain [10, Theorem 4(2)]. What makes all these examples work is the phenomenon that the property of being a $\Delta$-domain is not preserved by the $D+M$ construction. (Nelther is the QQR-property: see [10, Example 3].) Our final result will establish quite different behavior for CPI-closed domains. Besides this point of contrast, Proposition 3.11 also produces examples, with arbitrary Krull dimension exceeding 1 , of the behavior described in Remark 3.5 and Example 3.6. 
PROPOSITION 3.11. Let $K+M$ be a valuation domain with nonzero maximal ideal $M$, such that $K$ is a field. Let $D$ be a (proper) subring of $K$ - Then $D+M$ is CPI-closed if and only if $D$ is CPI-closed.

PROOF. As the primes of $R=D+M$ and the localizations of $R$ at those primes have been catalogued [8, Theorem A, p. 560], one readily verifies the following. If $P$ is a prime of $D$, the CPI-extension of $R$ with respect to $P+M$ is $E+M$, where $E$ is the CPI-extension of $D$ with respect to $P$; moreover, $R$ is the CPI-extension of $R$ with respect to each prime which is not of the form $P+M$. Since the " + " in $K+M$ is a direct sum, the conclusion now follows easily from Proposition 3.2.

Finally, observe that each of the following five types of rings is detectable locally: locally divided domain; going-down ring; treed domain; domain having the QQR-property (by [9, Theorem 1.9]); and $\Delta$-domain (by [10, Theorem 3]). However, as shown by Remark 3.5(b) and Example 3.6, being a CPI-closed domain is not a local property. We clòse by asking whether one can find an interesting new class of domains for which CPI-closedness would be detectable locally. One criterion for "interesting" might be that, as is the case with all the above classes, the new class be required to include all Prüfer domains.

NOTE ADDED IN PROOF (AUGUST 20, 1979). By reasoning as in the proofs of Proposition 2.3 and Theorem 2.4 , one sees that the following three conditions are equivalent for an integral domain $R$. (1) $R$ is locally divided and each nonzero prime ideal of $R$ lies in a unique maximal ideal of $R$; (2) For each nonzero $P \in \operatorname{Spec}(R)$, there exists a prime ideal $Q$ of $R$ such that $R+P R_{P}=R_{Q}$; (3) For each nonzero $P \in \operatorname{Spec}(R)$, there exists a (uniquely determined) maximal ideal $M$ of $R$ such that $R+P R_{P}=R_{M}$. 


\section{REFERENCES}

1. AKIBA, T. A note on AV-domains, Kyoto Univ. Education Ser. B, 31 (1967) 1-3.

2. BOISEN, M.B. Jr. and SHELdon, P.B. Pre-Prüfer rings, Pac. J. Math. $\underline{58}$ (1975) 331-344.

3. BOISEN, M.B., Jr. and SHELDON, P.B. CPI extensions: overrings of integral domains with special prime spectrums, Can. J. Math. 29 (1977) 722-737.

4. BOURBAKI, N. Commutative Algebra, Addison-Wesley, Reading, Mass., 1972.

5. DOBBS, D.E. On going down for simple overrings, II, Comm, in Algebra 1 (1974) 439-458.

6. DOBBS, D.E. Divided rings and going-down, Pac. J. Math. 67 (1976) 353-363.

7. DOBBS, D.E. Coherence, ascent of going-down, and pseudo-valuation domains, Houston J. Math. 4 (1978) 551-567.

8. GILMER, R.W. Multiplicative Ideal Theory, Queen's papers on pure and appl. math., Queen's University, Kingston, Ont. 12 (1968).

9. GILMER, R.W. and HEINZER, W.J. Intersections of quotient rings of an integra1 domain, J. Math. Kyoto Univ. I (1967) 133-150.

10. GILMER, R.W. and HUCKABA, J.A. $\Delta$-rings, J. A1gebra 28 (1974 ( 414-432.

11 GREENBERG, B. Global dimension of Cartesian squares, J. Algebra 32 (1974) 31-43.

12. PAPICK, I.J. Topologically defined classes of going-down domains, dissertation, Rutgers University, New Brunswick, N.J., 1975.

13. PAPICK, I.J. Topologically defined classes of going-down domains, Trans. Amer. Math. Soo. 219 (1976) 1-37.

14. Richman, R. Generalized quotient rings, Proc. Amer. Math. Soc. 16 (1965) 794-799. 


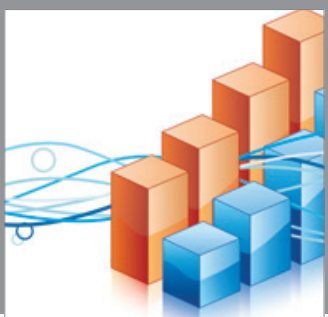

Advances in

Operations Research

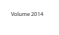

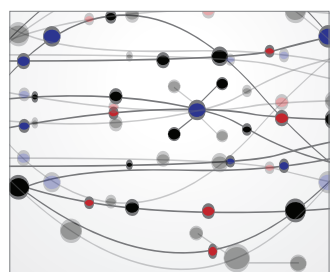

\section{The Scientific} World Journal
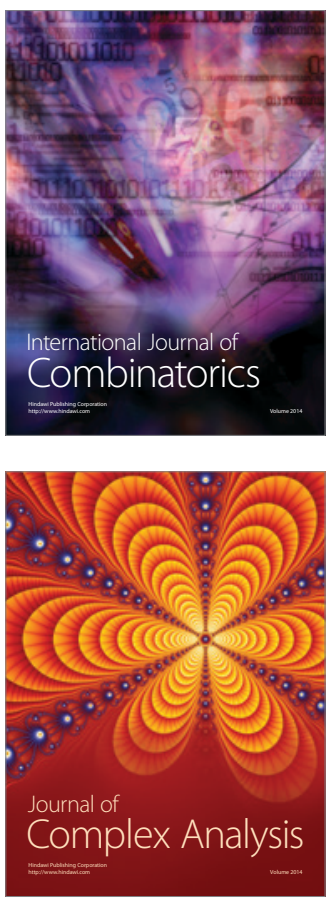

International Journal of

Mathematics and

Mathematical

Sciences
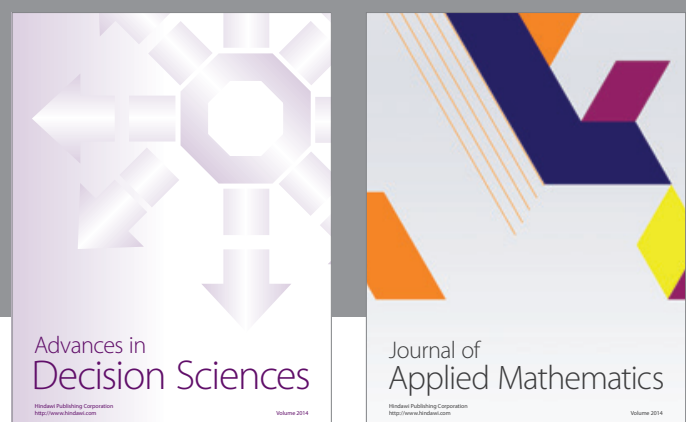

Journal of

Applied Mathematics
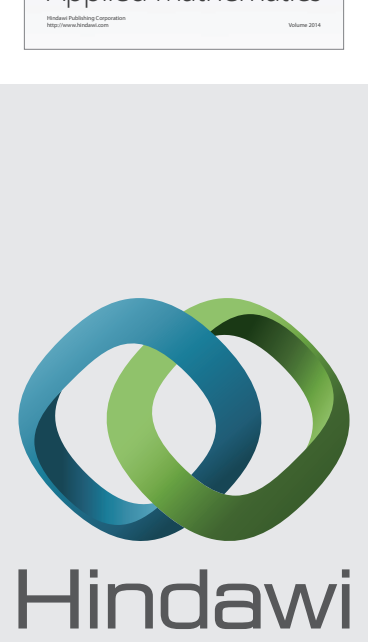

Submit your manuscripts at http://www.hindawi.com
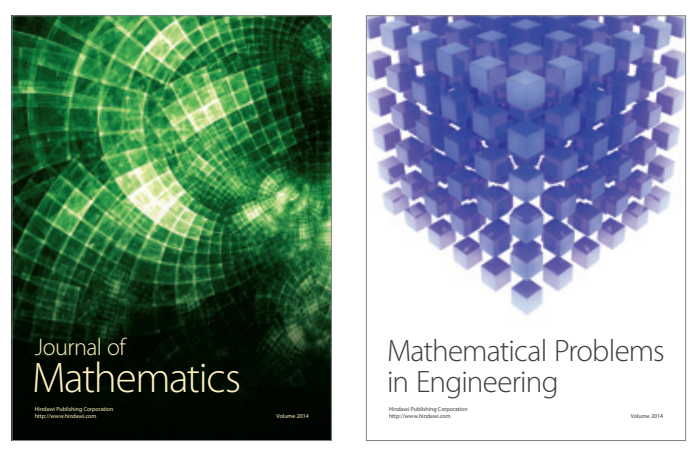

Mathematical Problems in Engineering
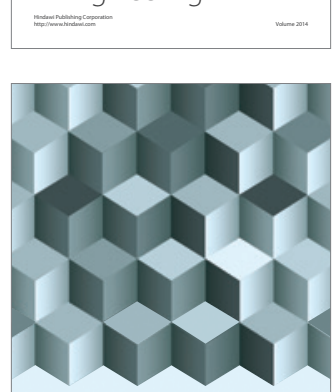

Journal of

Function Spaces
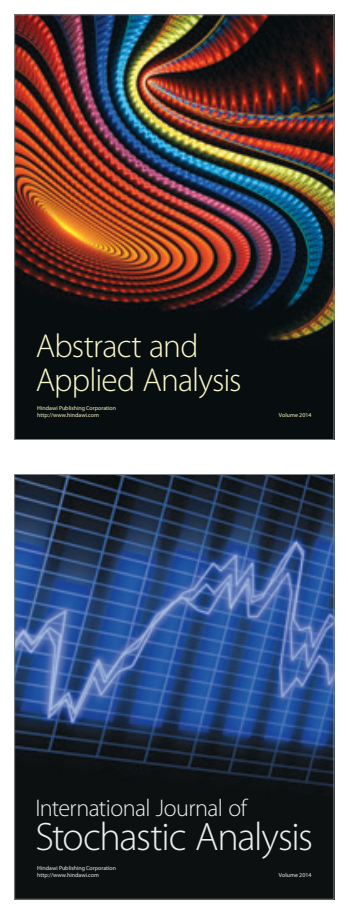

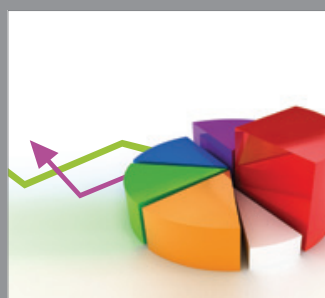

ournal of

Probability and Statistics

Promensencen
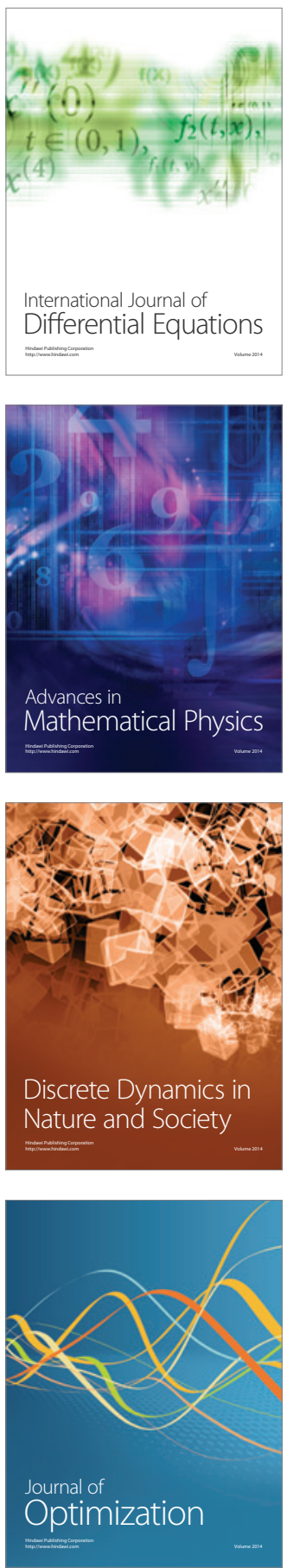